\title{
A random fiber bundle with many discontinuities in the threshold distribution
}

\author{
Uma Divakaran* and Amit Dutta ${ }^{\dagger}$ \\ Department of Physics, Indian Institute of Technology Kanpur - 208016, India
}

(Dated: June 1, 2018)

\begin{abstract}
We study the breakdown of a random fiber bundle model (RFBM) with $n$-discontinuities in the threshold distribution using the global load sharing scheme. In other words, $n+1$ different classes of fibers identified on the basis of their threshold strengths are mixed such that the strengths of the fibers in the $i-t h$ class are uniformly distributed between the values $\sigma_{2 i-2}$ and $\sigma_{2 i-1}$ where $1 \leq i \leq n+1$. Moreover, there is a gap in the threshold distribution between $i-t h$ and $i+1-t h$ class. We show that although the critical stress depends on the parameter values of the system, the critical exponents are identical to that obtained in the recursive dynamics of a RFBM with a uniform distribution and global load sharing. The avalanche size distribution (ASD), on the other hand, shows a non-universal, non-power law behavior for smaller values of avalanche sizes which becomes prominent only when a critical distribution is approached. We establish that the behavior of the avalanche size distribution for an arbitrary $n$ is qualitatively similar to a RFBM with a single discontinuity in the threshold distribution $(n=1)$, especially when the density and the range of threshold values of fibers belonging to strongest $(n+1)$-th class is kept identical in all the cases.

PACS numbers: 46.50. +a, 62.20.Mk, 64.60.Ht, 81.05.Ni
\end{abstract}

\section{INTRODUCTION}

What causes fracture of materials in nature? Are there any precursor that signals the imminence of a complete breakdown so that we can avoid them taking place? These are few of the many questions that physicists and engineers are looking at now a days to explore the fracture dynamics of heterogeneous materials 1, 2]. A prior knowledge of failure properties of such materials are of extreme importance for problems related to physics of breakdown, material science as well as architectural, mechanical and textile engineering. The simplest of all the attempts is a model of fibers with randomly distributed threshold strengths known as the random fiber bundle model.

In a random fiber bundle model (RFBM) 3, 4, 5, $6,7,8,9,10,11,12,13,14,15,16,17$, fibers with stochastically distributed values of threshold strengths (i.e., the maximum external stress a fiber can withstand) are clamped at both the ends. Since the threshold of a fiber depends crucially upon the presence of defects in that particular fiber, it is indeed useful to assign random threshold strength to each fiber of the bundle. The threshold strengths however are chosen from a given distribution, usually approximated by a Weibull or uniform distribution. Under the application of a weak external load, the fibers with threshold lying below the applied stress, break and the resulting additional load is distributed among the intact fibers using a load sharing rule. This redistribution of stress causes further failures and the dynamics stops when the system reaches a fixed point at which no further failure takes place. To resume

\footnotetext{
*Electronic address: udiva@iitk.ac.in
}

${ }^{\dagger}$ Electronic address: dutta@iitk.ac.in the recursive dynamics, the external load is further increased to break the next weakest intact fiber. The process continues until the complete breakdown of the entire bundle.

In this work, we shall use the global load sharing (GLS) rule where the additional load generated due to the breaking of a fiber is shared equally by all the intact fibers of the bundle. In the GLS scheme, the breakdown of the fiber bundle can be interpreted as a continuous phase transition with well defined critical stress and critical exponents [7, 8, 9, 10]. For a RFBM with GLS, it has also been established that the distribution $D(\Delta)$ of an avalanche of size $\Delta$, defined as the number of fibers broken between two successive loadings, satisfies a universal power-law $D(\Delta) \sim \Delta^{-5 / 2}$ in the limit of $\Delta \rightarrow \infty$ 4, 5]. In a recent work, Pradhan, Hansen and Hemmer 11] showed that in the vicinity of the critical distribution where the average external load on the bundle is maximum, the avalanche size distribution shows a crossover to a new power-law behavior given by $D(\Delta) \sim \Delta^{-3 / 2}$ for small values of $\Delta<<\Delta_{c}$ while for $\Delta>>\Delta_{c}$, the $\Delta^{-5 / 2}$ behavior is recovered. The characteristic size $\Delta_{c}$ around which the crossover occurs diverges in a power-law fashion as the critical distribution is approached and at the critical distribution the $\Delta^{-3 / 2}$ behavior is observed for the entire range of $\Delta$.

In a recent paper, the authors [15] investigated the robustness of the above universal power-law behavior of the avalanche size distribution by introducing a discontinuity in the threshold distribution $\rho\left(\sigma_{t h}\right)$ which is given by

$$
\begin{aligned}
\rho\left(\sigma_{t h}\right) & =\frac{1}{1-\left(\sigma_{2}-\sigma_{1}\right)} & & 0<\sigma_{t h} \leq \sigma_{1} \\
& =0 & & \sigma_{1}<\sigma_{t h}<\sigma_{2} \\
& =\frac{1}{1-\left(\sigma_{2}-\sigma_{1}\right)} & & \sigma_{2} \leq \sigma_{t h} \leq 1 .
\end{aligned}
$$

Hence, two types of fibers (weaker and stronger) sepa- 


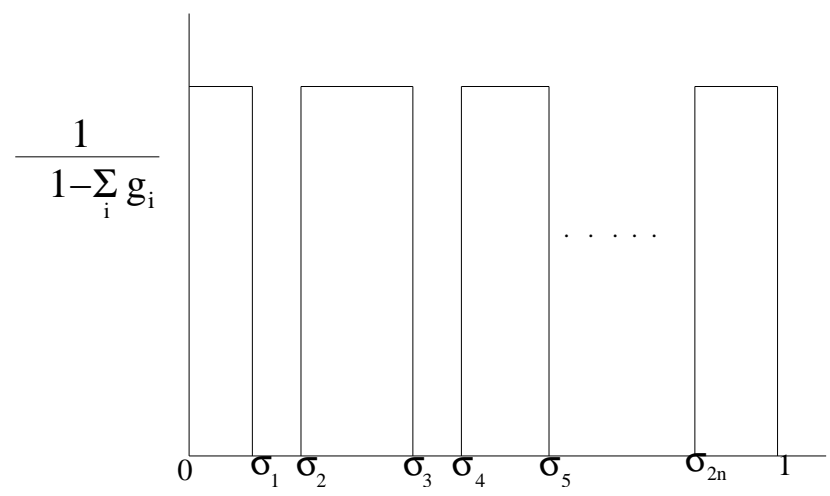

FIG. 1: Mixed Uniform Distribution with $(n+1)$ classes of fibers and $n$-gaps or discontinuities.

rated by a gap in their threshold distributions coexist in the same bundle with more than half of the fibers belonging to the stronger class. It has been established that there exists a non-universal non-power law behavior in the avalanche size distribution for small $\Delta$ which crosses over to the universal behavior $\Delta^{-5 / 2}$ in the asymptotic limit of $\Delta$. Most interestingly, it was pointed out that the non-universality becomes prominent only when a critical distribution is approached. The threshold distribution given in Eq. 1 with $\sigma_{2}=0.5$ is the critical distribution of a RFBM with single discontinuity [15], because as soon as the redistributed stress reaches 0.5 , the bundle is critical and breaks down completely with an infinitesimal increase in the external load. At a critical distribution, however, there is a crossover from the non-universal behavior to a power-law behavior with $\Delta^{-3 / 2}$ for large $\Delta$. A recent study of an infinite gap generalization of the above model predicts a new exponent $(=9 / 4)$ of the avalanche size distribution [17].

The natural question that remains is what would be the effect of many such discontinuities on the avalanche size distribution? We address this issue in the present communication where we study a RFBM with $n$-discontinuities in the threshold distribution and investigate its effect on the critical behavior and the avalanche size distribution. The paper is organized as follows: In Section II, we introduce the model and derive the critical stress and exponents using the recursive dynamics approach. The results on the avalanche size distribution and comparison with the $n=1$ case studied previously are presented in section III. We make concluding comments in section IV.

\section{THE MODEL AND THE RECURSIVE DYNAMICS}

The threshold distribution of a RFBM with $n$ discontinuities studied in this paper is shown in Fig. 1. The threshold range of the weakest and the strongest class of fibers is from 0 to $\sigma_{1}$ and $\sigma_{2 n}$ to 1 , respectively.
The mathematical form of the normalized threshold distribution is given by

$$
\begin{aligned}
\rho\left(\sigma_{t h}\right) & =\frac{1}{1-\sum_{i=1}^{n}\left(\sigma_{2 i}-\sigma_{2 i-1}\right)} \\
& =\quad \frac{1}{1-\sum_{i=1}^{n} g_{i}} \text { for } \sigma_{2 i-2}<\sigma_{t h} \leq \sigma_{2 i-1}(2) \\
& =0 \quad \text { otherwise }
\end{aligned}
$$

where $1 \leq i \leq n+1, g_{i}=\sigma_{2 i}-\sigma_{2 i-1}$ and $\sigma_{0}=0, \sigma_{2 n+1}=$ 1. The above distribution suggests that $(n+1)$ different classes of fibers with the threshold of the fibers of the $i$-th class ranging from $\sigma_{2 i-2}$ to $\sigma_{2 i-1}$ are mixed. At the same time the restriction $0<\sigma_{1}<\sigma_{2} \ldots<\sigma_{2 i-1}<\sigma_{2 i}<1$ ensures the existence of a gap or discontinuity given by $g_{i}$ in the threshold strengths between $i$-th and $(i+1)$-th class of fibers. We also assume that the threshold values of the $i$-th class of fibers are uniformly distributed within the range $\sigma_{2 i-2}$ to $\sigma_{2 i-1}$ for all $i$, a condition that leads to a set of $(n+1)$ additional relations connecting the parameters of the system in the following way. Distributing a fraction $f_{i}$ of total number of fibers to the $i$-th class (with $\sum_{i}^{n+1} f_{i}=1$ ) and using the uniformity condition mentioned above we get

$f_{i}=\frac{\sigma_{2 i-1}-\sigma_{2 i-2}}{1-\sum_{i=1}^{n} g_{i}}$

such that $\sigma_{0}=0, \sigma_{2 n+1}=1$ and once again $1 \leq i \leq n+1$ The model therefore involves $(2 n)$ values of $\sigma_{i}$ 's, $(n+1)$ values of density $f_{i}$, the conditions given by Eq. 4 along with the additional restrictions $\sum_{i}^{n+1} f_{i}=1$. Therefore, the total number of free variables that can be chosen independently reduces to $2 n$. It should also be noted that since $1-\sum_{i=1}^{n} g_{i}$ is always less than unity, we must have $\left(\sigma_{2 i-1}-\sigma_{2 i-2}\right)<f_{i}$ for all $i$.

We shall estimate the critical stress of the above model within the framework of a recursive dynamics and global load sharing. The fibers belonging to all $(n+1)$-classes cooperatively participate in sharing the additional load arising due to the breaking of the weaker fibers. The fraction of unbroken fibers after a time step $t+1$, denoted by $U_{t+1}$, is related to $U_{t}$ through the relation [10, 15]

$U_{t+1}=1-P\left(\sigma_{t}\right)=1-P\left(\frac{\sigma}{U_{t}}\right)$

where $P\left(\sigma_{t}\right)$ is the fraction of broken fibers with the applied stress $\sigma$ and redistributed stress $\sigma_{t}$, and is given as

$$
P\left(\sigma_{t}\right)=\int_{0}^{\sigma_{t}} \rho\left(\sigma_{t h}\right) d \sigma_{t h}
$$

Similarly, the redistributed stress after a time step $(t+1)$ satisfy the recursive relation

$\sigma_{t+1}=\frac{\sigma}{U_{t+1}}=\frac{\sigma}{\left(1-P\left(\sigma_{t}\right)\right)}$ 
The fixed point solution for $U\left(=U^{*}\right)$ at which no further failure takes place can be obtained by solving the above recursive relations (5) and (6) [10]. Assuming that the redistributed stress at some instant $t, \sigma_{t}$ exceeds $\sigma_{2 n}$ (i.e, when the redistributed stress initiates the breaking of the $(n+1)$-th class fibers), we obtain

$$
\begin{aligned}
U_{t+1} & =1-P\left(\frac{\sigma}{U_{t}}\right) \\
& =1-\left[\sum_{i=1}^{n} \frac{\sigma_{2 i-1}-\sigma_{2 i-2}}{1-\sum_{i}^{n} g_{i}}+\frac{1}{1-\sum_{i}^{n} g_{i}}\left(\frac{\sigma}{U_{t}}-\sigma_{2 n}\right)\right]
\end{aligned}
$$

so that we get the fixed point solution

$U^{*}=\frac{1}{2\left(1-\sum_{i}^{n} g_{i}\right)}\left[1+\sqrt{1-\frac{\sigma}{\sigma_{c}}}\right]$

Along the same line of arguments, we find the redistributed stress at the fixed point

$\sigma^{*}=\frac{1}{2}-\frac{1}{2} \sqrt{1-\frac{\sigma}{\sigma_{c}}}$.

Using Esq. (7) and (8) we find that the critical stress is given by

$\sigma_{c}=\frac{1}{4\left[1-\left(\sum_{i}^{n} g_{i}\right)\right]}$.

which is the applied load per fiber at which half of the fibers break. When the external load exceeds $\sigma_{c}$ even by an infinitesimal amount, there is no real solution of $U^{*}$ and $\sigma^{*}$ which signals the complete break down of the bundle.

The critical stress of the mixed model varies with the gaps $g_{i}$ and in the limit $g_{i} \rightarrow 0$ for each $i$, we retrieve the critical stress $\sigma_{c}=1 / 4$ for a RFBM with uniform distribution [10]. We also get back the result of single discontinuity case i.e., $n=1[15]$ if only $g_{1} \neq 0$. The equation (8) also shows that the redistributed stress attains the maximum value of 0.5 at the critical external stress $\sigma_{c}$.

Calling the redistributed stress as $\mathrm{x}$ from now onwards, we consider the constitutive equation, $F(x)=$ $N x(1-P(x)$ where $F(x)$ is the average external load when the redistributed stress is $x$. The load $F(x)$ maximizes when the redistributed stress $x$ is equal to 0.5 . Since the maximum value of the redistributed stress is equal to $0.5, \sigma_{2 n}$ must be less than 0.5 so that some fibers from $(n+1)$-th class also fail at the critical point. The constraint equations (4) suggest that for recursive dynamics to hold good, more than half of the fibers must belong to the $(n+1)$-th class with thresholds lying between $\sigma_{2 n}$ to 1 . We therefore define the threshold distribution $\rho\left(\sigma_{t h}\right)$ (Eq. 2), along with the constraint conditions and $\sigma_{2 n}=0.5$ as the critical distribution. It can also be shown in a straightforward way that the order parameter exponent $(\beta)$ and the susceptibility exponents $(\gamma)$ stick to the mean field (GLS) values with $\beta=\gamma=1 / 2$, even in the presence of an arbitrary number of discontinuities provided uniformity condition is satisfied and hence the critical behavior remains unaltered although there is an appreciable change in the critical stress.

\section{AVALANCHE SIZE DISTRIBUTION (ASD)}

We shall now turn our attention towards the ASD of RFBM in the presence of many discontinuities in the threshold distribution which is the key point of our study. We show below that the discontinuities have a non-trivial affect on the ASD when a critical distribution is approached. We also argue that a situation with many discontinuities is qualitatively similar to the singlediscontinuity case. The scenario is established considering a special case with $n=2$, where $g_{1}=\sigma_{2}-\sigma_{1}$ and $g_{2}=\sigma_{4}-\sigma_{3}$ so that three classes of fibers with range of thresholds lying between ( 0 to $\left.\sigma_{1}\right),\left(\sigma_{2}\right.$ to $\left.\sigma_{3}\right)$ and ( $\sigma_{4}$ to 1 ), respectively, coexist in the bundle while $f_{1}, f_{2}$ and $f_{3}$ are the corresponding densities.

Below is shown some of the allowed distributions which satisfy the restrictions mentioned in Eq. 4 and case 4 refers to a critical distribution.

\begin{tabular}{|c|c|c|c|c|c|c|r|}
\hline Case & $f_{1}$ & $f_{2}$ & $\sigma_{1}$ & $\sigma_{2}$ & $\sigma_{3}$ & $\sigma_{4}$ & $\sigma_{c}$ \\
\hline 1 & 0.10 & .20 & 0.08 & 0.16 & 0.32 & 0.44 & 0.31 \\
\hline 2 & 0.15 & 0.25 & 0.135 & 0.15 & 0.375 & 0.46 & 0.27 \\
\hline 3 & 0.05 & 0.05 & 0.04 & 0.16 & 0.20 & 0.28 & 0.31 \\
\hline 4 & 0.10 & 0.20 & 0.07 & 0.16 & 0.30 & 0.50 & 0.35 \\
\hline 5 & 0.10 & 0.2 & 0.08 & 0.26 & 0.42 & 0.44 & 0.31 \\
\hline 6 & 0.20 & 0.15 & 0.16 & 0.2 & 0.32 & 0.48 & 0.31 \\
\hline
\end{tabular}

We shall now generalize the results of Hemmer and Hansen [4] to study the avalanche behavior of the $n=$ 2-discontinuity model. The general expression for the avalanche size distribution with GLS is given as

$$
\begin{array}{rl}
\frac{D(\Delta)}{N}=\frac{\Delta^{\Delta-1}}{\Delta !} \int_{0}^{x_{c}} & d x \rho(x) \frac{(1-a(x))}{a(x)} \\
+ & \times \exp (\{-a(x)+\ln a(x)\} \Delta)
\end{array}
$$

where $x$ is the redistributed stress and the upper limit of the integration $\left(x_{c}\right)$ is the redistributed stress at the critical point. Also, $a(x)=x \rho(x) / 1-P(x)$ is the number of fibers that break as a result of breaking a fiber with threshold strength $x$ by applying an external load $x /\{(1-$ $P(x)) N\}$.

Let us first consider the breaking of fibers belonging to class 1 with threshold values uniformly lying between 0 to $\sigma_{1}$, this contribution $D_{1}(\Delta)$ is given by

$$
\begin{array}{rl}
D_{1}(\Delta)=\frac{\Delta^{\Delta-1}}{\Delta !} \int_{0}^{\sigma_{1}} & d x \frac{1}{1-g_{1}-g_{2}} \frac{(1-a(x))}{a(x)} \\
& \times \exp (\{-a(x)+\ln a(x)\} \Delta)
\end{array}
$$

where $a(x)=x /\left(1-g_{1}-g_{2}-x\right)$. The maximum contribution of this integral is at $a(x)=1$ or $x=\left(1-g_{1}-g_{2}\right) / 2$ which exceeds $\sigma_{1}$, i.e., lies beyond the range of integration and we can not employ the method of the saddle point integration. However, $a(x)$ is a monotonically increasing function of $x$ up to $\sigma_{1}$ and we therefore get the 


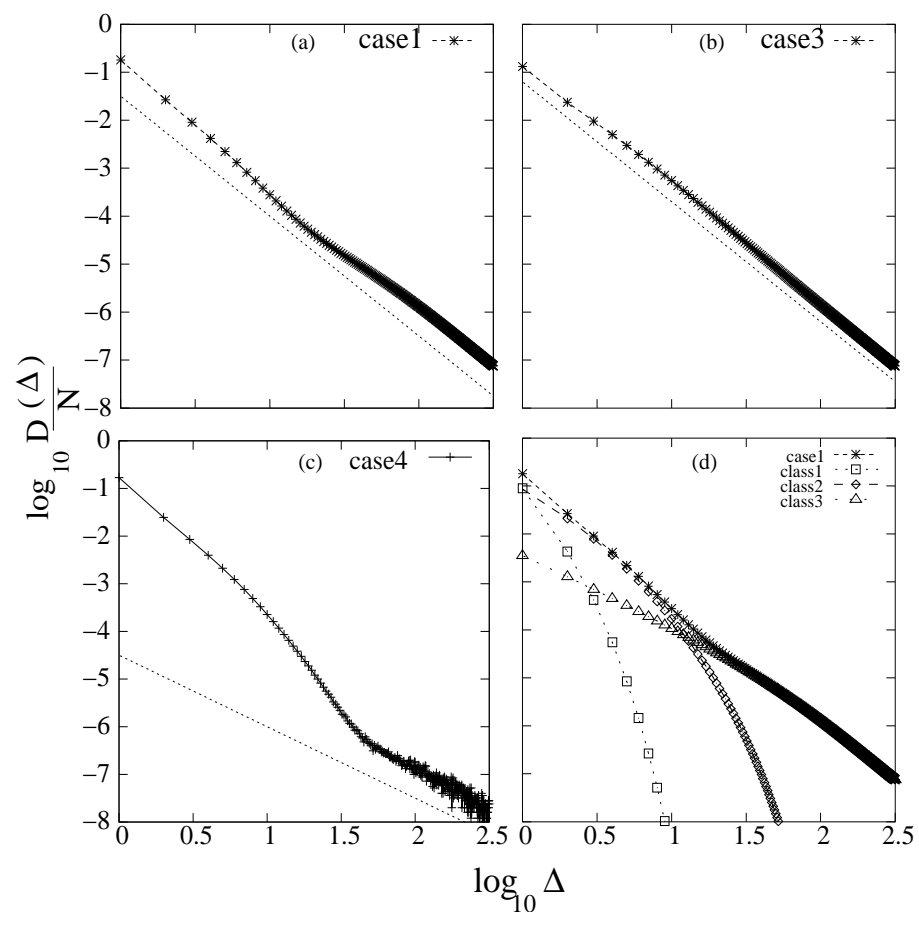

FIG. 2: Fig. (2a) corresponds to the ASD for case1 of the table where a crossover from nonuniversal to the universal $5 / 2$ behavior is observed since $\sigma_{4}$ is close to 0.5 . (2b) shows case 3 of the table where no non-universal behavior is observed except for small values of $\Delta$. ASD for a critical distribution (case4) is shown in Fig. 2(c) where there is a crossover to the " $3 / 2$ " behavior for large $\Delta$. The dotted line in Fig $2 \mathrm{a}$ and $2 \mathrm{~b}$ has a slope $-5 / 2$ whereas that in Fig $2 c$ has a slope $-3 / 2$. In $2(\mathrm{~d})$, we show $D_{1}(\Delta), D_{2}(\Delta), D_{3}(\Delta)$ and the total ASD for the case 1 to compare their relative magnitudes.

maximum contribution when $x$ reaches the upper limit of the integration $\sigma_{1}$. The leading behavior of $D_{1}(\Delta)$ (with $\left.\Delta !=\exp (-\Delta) \Delta^{\Delta} \sqrt{2 \pi \Delta}\right)$, is given by

$D_{1}(\Delta) \sim \frac{1}{\sqrt{2 \pi}\left(1-g_{1}-g_{2}\right)} \Delta^{-5 / 2} x_{m}^{\Delta} \exp \left(\left(1-x_{m}\right) \Delta\right),(12$

where $x_{m}=\sigma_{1} /\left(1-g_{1}-g_{2}-\sigma_{1}\right)=f_{1} /\left(1-f_{1}\right)$. Therefore, $D_{1}(\Delta)$ exhibits a non-universal decay with increasing $\Delta$ which is more rapid if $f_{1} \rightarrow 0$.

Similarly, the leading contribution of the fibers belonging to the class 2 with threshold values ranging from $\sigma_{2}$ to $\sigma_{3}$ and $a(x)=x /\left(1-g_{2}-x\right)$ is found to be

$D_{2}(\Delta) \sim \frac{1}{\sqrt{2 \pi}\left(1-g_{1}-g_{2}\right)} \Delta^{-5 / 2} y_{m}^{\Delta} \exp \left(\left(1-y_{m}\right) \Delta\right)$,

where $y_{m}=\sigma_{3} /\left(1-\sigma_{4}\right)$. Therefore, $D_{2}(\Delta)$ shows a similar non-universal behavior which survives even for relatively higher values of $\Delta$ if $\sigma_{3}$ approaches $\sigma_{4}$ and also $\sigma_{4} \rightarrow 0.5$. The significance of the above findings is explained below.

Let us now focus on the contribution from the fibers belonging to class 3 . Using Eq. (10) with $a(x)=x /(1-x)$ and $f_{3}=\left(1-\sigma_{4}\right) /\left(1-g_{1}-g_{2}\right)$, we get

$$
\begin{aligned}
D_{3}(\Delta)=\frac{1}{\sqrt{2 \pi \Delta}} & \frac{f_{3}}{1-\sigma_{4}} \int_{\sigma_{4}}^{0.5} d x \frac{(1-2 x)}{x} \\
& \times \exp \left(\left\{-\frac{x}{1-x}+\ln \left(\frac{x}{1-x}\right)\right\} \Delta\right)
\end{aligned}
$$

Right hand side of Eq. (14) can be integrated to obtain,

$D_{3}(\Delta) \sim \frac{f_{3}}{2 \sqrt{2 \pi} \Delta^{5 / 2}\left(1-\sigma_{4}\right)}\left(1-e^{-\frac{\Delta}{\Delta_{c}}}\right)$

where $\Delta_{c} \sim\left(1 / 2-\sigma_{4}\right)^{-2}$, which diverges in the limit $\sigma_{4} \rightarrow 0.5$. Our observations are depicted in Fig. 2, where $D(\Delta)$, obtained by numerical integration and also by simulation using the weakest fiber approach [4], is plotted for different threshold distributions. It is to be noted that the contribution $D_{3}(\Delta)$ depends only on $\sigma_{4}$ and $f_{3}$, an observation that leads to an interesting conclusion that the contribution to the total avalanche size distribution coming from the strongest class of fibers is identical for any number of discontinuities if the fraction of fibers as well as the range of the threshold strength of the final block is kept fixed (see Fig. 3). Equation (15) provides two limiting power-law behaviors given as

$$
\begin{aligned}
D_{3}(\Delta) & \sim \Delta^{-3 / 2} \text { for } \Delta<\Delta_{c} \\
& \sim \Delta^{-5 / 2} \text { for } \Delta>\Delta_{c}
\end{aligned}
$$

Comparing Eqs. (12), (13) and (16), we observe that in the limit of small $\Delta$, the non-power law contributions from $D_{1}(\Delta)$ and $D_{2}(\Delta)$ dominates over the universal " $5 / 2$ " behavior only in the limit of $\sigma_{4} \rightarrow 0.5$ when $D_{3}(\Delta) \sim \Delta^{-3 / 2}$. Otherwise, $D_{3}(\Delta)$ dominates over the non-universal contributions so that one observes a universal behavior nearly for the entire range of $\Delta$ though the discontinuities in the distribution always exist. At a critical distribution however, there is a crossover to the universal behavior $D(\Delta) \sim \Delta^{-3 / 2}$ for very large $\Delta$ following a large region of non-universality (see Fig. 2c). This general behavior is valid for any number of discontinuities including $n=1$ [15].

Let us now concentrate on some interesting limiting situations to investigate the role of two discontinuities: (i) If the fraction $f_{1}$ of fibers in class 1 is small, the contribution $D_{1}(\Delta) \sim\left[f_{1} /\left(1-f_{1}\right)\right]^{\Delta}$ dies off rapidly. However, if $\sigma_{3}$ is large and $\sigma_{4}$ approaches 0.5 , there is a wide region of non-universality in $(D(\Delta)-\Delta)$ behavior which is solely due to $D_{2}(\Delta)$ which scales as $\left[\sigma_{3} /\left(1-\sigma_{4}\right)\right]^{\Delta}$. (ii) In the other limit, when $f_{2}<f_{1}$ (but $f_{2}+f_{1}<0.5$, as required), $D_{1}(\Delta)$ do dominate in the small $\Delta$ limit, but for large $\Delta$ once again it is the contribution of $D_{2}(\Delta)$, rather the larger value of $\sigma_{3}$ that leads to a prominent non-universal behavior, e.g., case 6 in table 1. We therefore conclude that the contribution from the weakest class of fibers is not significant in the large $\Delta$ limit and it is the higher value of $y_{m}=\sigma_{3} /\left(1-\sigma_{4}\right)$ that causes the non-universality to survive up to relatively higher values of $\Delta$ (Fig. 4) 


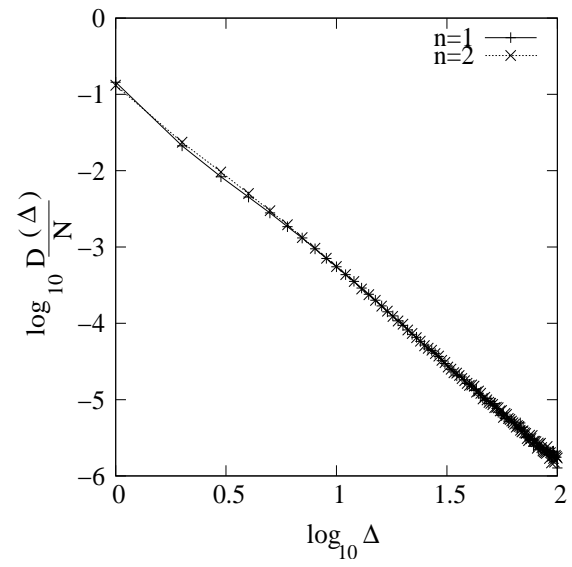

FIG. 3: Comparison of total avalanche size distribution for same value of $f_{n+1}$ and $\sigma_{2 n}$ when $n=1$ and $n=2$ where $n$ is the number of discontinuities. Clearly, the two cases overlap in the large $\Delta$ region where the final block dominates. Here, $f_{n+1}=0.9$ and $\sigma_{2 n}=0.28$

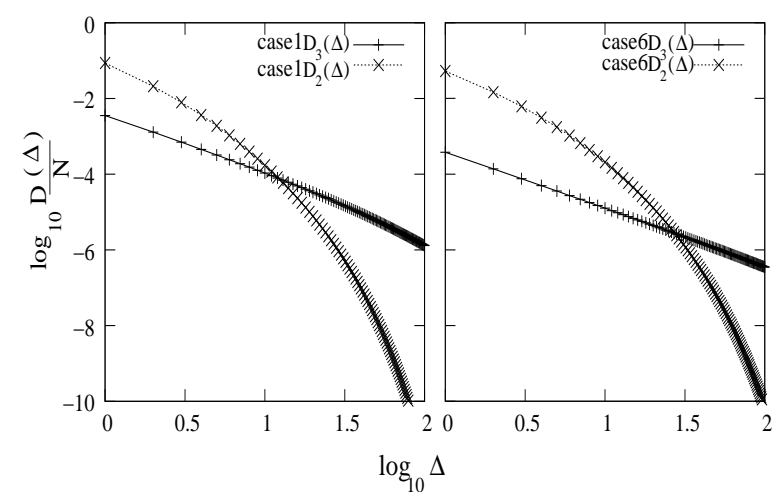

FIG. 4: The figure shows the comparison of $D_{2}(\Delta)$ with $D_{3}(\Delta)$ for case 1 (left panel) and case 6 (right panel). The figure shows that the crossover to universal behavior occurs at higher value of $\Delta$ if $y_{m}=\sigma_{3} /\left(1-\sigma_{4}\right)$ increases.

\section{CONCLUSIONS}

In conclusion, we have studied a mixed fiber bundle with many discontinuities in the threshold distribution and GLS where threshold values of the fibers belonging to a particular class are uniformly distributed within the specified range. Our studies lead to the following conclusions for an arbitrary number of discontinuities: (i) The recursive dynamics studies point to the existence of a critical distribution as defined in the text. (ii) There exists a non-universal, non- power law behavior in the avalanche size distribution for small $\Delta$ which becomes prominent only when a critical distribution is approached, otherwise it is masked by the universal behavior except for very small $\Delta$. For asymptotically large $\Delta$, however, there is always a crossover to the universal behavior with $\xi=5 / 2$ (or $\xi=3 / 2$ at the critical distribution). The crossover occurs around $\Delta=\Delta_{c}$ where the contribution from the strongest class of fibers $D_{n+1}(\Delta)$ switches from $\xi=3 / 2$ to $\xi=5 / 2$ behavior. (iii) $D_{n+1}(\Delta)$ is found to depend only on $f_{n+1}$ and $\sigma_{2 n}$ so the contribution of the fibers belonging the strongest class (i.e., the behavior of total $D(\Delta)$ in the limit of large $\Delta$ ) remains identical for any number of discontinuities if $f_{n+1}$ and $\sigma_{2 n}$ are kept fixed. (iv) We also show that if $\sigma_{2 n-1}$ increases and at the same time $\sigma_{2 n} \rightarrow 0.5$, the non-universality survives up to higher values of $\Delta$.
[1] B. K. Chakrabarti and L. G. Benguigui, Statistical Physics of fracture and Breakdown in Disordered Systems, Oxford Univ. Press, Oxford (1997); M. Sahimi, Heterogeneous Materials II: Nonlinear Breakdown Properties and Atomistic Modelling, Springer-Verlag Heidelberg, (2003); H. J. Herrmann and S. Roux, Statistical Models of Disordered Media, North Holland, Amsterdam (1990).

[2] R. L. Smith, Proc. R. Soc. London A 372, 539 (1980); S. Zapperi, P. Ray, H. E. Stanley, A. Vespignani, Phys. Rev. Lett. 78, 1408 (1997); J. V. Andersen, D. Sornette and K. T. Leung, Phys. Rev. Lett 78, 2140 (1997); S. D. Zhang and E-jiang Ding, Phys. Rev. 53, 646 (1996); B. Q. Wu and P. L. Leath, Phys. Rev. B 59, 4002 (1999).

[3] F. T. Peirce, J. Text. Inst. 17, 355 (1926); H. E. Daniels, Proc. R. Soc. London A 183404 (1945); B. D. Coleman, J. Appl. Phys. 29, 968 (1958); R. L. Smith, Proc. R. Soc. London A 372, 539 (1980); R. da Silveria, Am. J. Phys.
67, 1177 (1999);

[4] P. C. Hemmer and Alex Hansen, J. Appl. Mech. 59, 909 (1992); A. Hansen and P. C. Hemmer, Trends in Stat. Phys. 1, 213 (1994); A. Hansen and P. C. Hemmer, Phys. Lett. A 184, 394 (1994).

[5] M. Kloster, A. Hansen and P. C. Hemmer, Phys. Rev. E 56, 2615 (1997).

[6] J. B. Gomez, D. Iniguez and A. F. Pacheco, Phys. Rev. Lett. 71, 380 (1993).

[7] J. V. Andersen, D. Sornette, and K. -T. Leung, Phys. Rev. Lett. 78, 2140 (1997); D. Sornette and J. V. Andersen, Eur. Phys. J. B 1, 353 (1998).

[8] Y. Moreno, J. B. Gomez and A. F. Pacheco, Phys. Rev. Lett. 85, 2865 (2000).

[9] S. Pradhan and B. K. Chakrabarti, Int. J. Mod. Phys. B 17, 5565 (2003); P. C. Hemmer, A. Hansen and S. Pradhan, e-print cond-mat/0602371, in Modelling Critical and Catastrophic Phenomena in Geoscience, edited 
by P. Bhattacharya and B. K. Chakrabarti (Springer, Berlin, 2006). p. 27.

[10] S. Pradhan, P. Bhattacharyya and B.K. Chakrabarti, Phys. Rev. E 66, 016116 (2002); P. Bhattacharyya, S. Pradhan and B.K. Chakrabarti, Phys. Rev. E 67, 046122 (2003).

[11] S. Pradhan, A. Hansen and P. C. Hemmer, Phys. Rev. Lett. 95, 125501 (2005); S. Pradhan, A. Hansen and P. C. Hemmer, Phys. Rev. E 74016122 (2006); S. Pradhan and A. Hansen, Phys. Rev. E. 72, 026111 (2005).

[12] D. -H. Kim, B. J. Kim and H. Jeong, Phys. Rev. Lett.94, 025501 (2005); U. Divakaran and A. Dutta, Int. J. Mod. Phys C, 18919 (2007)

[13] F. Raischel, F. Kun and H. J. Herrmann, Phys. Rev. E
74, 035104(R) (2006); F. Kun and S. Nagy, Phys. Rev. E 77, 016608 (2008).

[14] Uma Divakaran and Amit Dutta, Phys. Rev. E 75011109 (2007).

[15] Uma Divakaran and Amit Dutta, Phys. Rev. E, 75 011117 (2007).

[16] S. Pradhan and Per C. Hemmer, Phys. Rev. E 77, 031138 (2008); S. Pradhan and Per C. Hemmer, Phys. Rev. E 75, 056112 (2007); Per C. Hemmer and S. Pradhan, Phys. Rev. E 75, 046101 (2007).

[17] R. C. Hidalgo, K. Kovacs, I. Pagonabarraga and F. Kun, Eur. Phys. Lett., 8154005 (2008). 